and KEGG biological pathway analysis were performed to predict the functions of differentially expressed IncRNAs and co-expressed potential targeting genes. Subsequently, a ceRNA (IncRNA-miRNA-mRNA) network including 2320 ceRNA pairs was constructed based on predicted miRNAs shared by IncRNAs and mRNAs.

Conclusion: The expression profile provided a systematic perspective on the potential functions of IncRNAs miRNAs, mRNAs and circRNAs in the pathogenesis of pSS. Therefore, this study will aid in the development of new diagnostic biomarkers and drug therapies.

REFERENCES:

[1] Le Dantec C, Varin MM, Brooks WH, Pers JO, Youinou P, Renaudineau

Y. Epigenetics and Sjogren's syndrome.Curr Pharm Biotechnol. 2012 Aug;13(10):2046-53.

Disclosure of Interests: None declared

DOI: 10.1136/annrheumdis-2021-eular.3784

\section{POS0786 UNMET TREATMENT NEEDS IN SYSTEMIC LUPUS ERYTHEMATOSUS (SLE): A CROSS-SECTIONAL ASSESSMENT OF DISEASE ACTIVITY IN SLE PATIENTS DURING THEIR LAST VISIT}

O. Gioti $^{1}$, K. Chavatza ${ }^{2}$, G. Belevonis ${ }^{1}$, S. Koutsoviti ${ }^{1}$, C. Katsimpari ${ }^{1}$, I. Sofianos ${ }^{1}$, A. Tatsi ${ }^{1}$, P. Tzanis ${ }^{1}$, A. Lazarini ${ }^{1}$, K. Klavdianou ${ }^{1}$, I.

I. Giannakopoulou ${ }^{1}$, E. Kalavri ${ }^{1}$, E. Theotikos ${ }^{1}$, A. Elezoglou ${ }^{1}$, D. Boumpas ${ }^{2}$, A. Fanouriakis 1,2 . "'Asklepieio"General Hospital, Department of Rheumatology, Athens, Greece; " "Attikon" University Hospital, Rheumatology and Clinical Immunology Unit, Athens, Greece

Background: The current goal of treatment in SLE is remission or low disease activity (LDA) and prevention of flares, achieved with the lowest possible dose of glucocorticoids. Nevertheless, in current clinical practice a significant number of patients still has active disease. ${ }^{1,2}$

Objectives: To assess the current disease activity state of SLE patients during their most recent visit in two centers (Department of Rheumatology in "Asklepieio" Hospital and Rheumatology Unit in "Attikon" Hospital, both in Athens, Greece).

Methods: Cross-sectional study of patients with a diagnosis of SLE for at least one year. Patients were divided into four groups: 1) Remission off-therapy: SLE Disease Activity Index (SLEDAI)=0 without prednisone or immunosuppressive drugs (IS), 2) Remission on-therapy: SLEDAI=0, prednisone dose $\leq 5 \mathrm{mg} /$ day and/or IS (conventional and biologic, maintenance phase), 3) LDA: SLEDAI $\leq 4$, prednisone dose $\leq 7.5 \mathrm{mg} /$ day and/or IS (maintenance phase), 4) Active disease: SLEDAI $>4$ and/or prednisone dose $>7.5 \mathrm{mg} /$ day and/or IS (induction phase). ${ }^{2}$ Hydroxychloroquine was allowed in all groups.

Results: 205 patients were included [95.1\% female, mean (SD) age 48.4 (14.9) years and median disease duration (IQR) 6.2 (12.6) years]. A history of lupus nephritis and neuropsychiatric SLE was present in $16.6 \%$ and $17.1 \%$ of our patients, respectively, and $39 \%$ of patients had SLICC/ACR damage index (SDI) $>0$. At last visit, remission off-therapy and remission on-therapy was present in $8.3 \%(n=17)$ and $15.1 \%(n=31)$ of our patients, respectively. Seventy-five patients $(36.6 \%)$ had LDA, whereas 82 patients $(40 \%)$ had active disease. More than $85 \%(86.3 \%)$ of patients were in treatment with hydroxychloroquine and $64.4 \%$ were receiving immunosuppressive drugs. Regarding glucocorticoids, $50.2 \%(n=103)$ were treated with prednisone dose $\leq 7.5 \mathrm{mg} /$ day and over $40 \%(42.4 \%, \mathrm{n}=87)$ did not receive prednisone at all. A SLEDAI score 0 and $1-4$ was achieved in $24.4 \%$ and $42.9 \%$ of patients, respectively, but only $3.9 \%$ had a SLEDAI $>8$, indicative of high disease activity. Conclusion: Although the majority of our patients were treated with hydroxychloroquine and glucocorticoids in acceptable levels of daily dose, four out of ten patients in our practice have active disease during their last visit. Achieving treatment goals in SLE patients remains a challenge for future novel therapies. REFERENCES:

[1] Fanouriakis A, Kostopoulou M, Alunno A, et al. 2019 update of the EULAR recommendations for the management of systemic lupus erythematosus. Ann Rheum Dis 2019; 78: 736-745.

[2] Ugarte-Gil MF, Wojdyla D, Pons-Estel GJ, et al. Remission and Low Disease Activity Status (LDAS) protect lupus patients from damage occurrence: data from a multiethnic, multinational Latin American Lupus Cohort (GLADEL). Ann Rheum Dis 2017; 76: 2071-2074.

Disclosure of Interests: None declared

DOI: 10.1136/annrheumdis-2021-eular.3832

\section{POS0787 BERBERINE MODULATE LUPUS SYNDROME VIA THE REGULATION OF GUT MICROBIOTA IN MRL/LPR MICE}

$\underline{\mathrm{Y} . ~ B a o}^{1}, \mathrm{J.} \mathrm{Ji}^{1}$, Z. Xue ${ }^{2}$, Z. Gu${ }^{1} .{ }^{1}$ Affiliated Hospital of Nantong University, Department of Rheumatology, Nantong, China; ${ }^{2}$ Affiliated Hospital of Nantong University, Research Center of Clinical Medicine, Nantong, China

Background: Intestinal flora disorder and immune abnormalities have been reported in systemic lupus erythematosus (SLE) patients ${ }^{1,2}$. Berberine (BBR) showed significant effects in regulating the intestinal flora, repairing gut barriers and regulating immune cells ${ }^{3,4}$. While few reports mentioned the abnormal gut microbiota and metabolites in Chinese SLE patients.

Objectives: Our investigation tried to illustrate the relationship between gut microbiota, intestinal metabolites and disease activity in Chinese SLE patients. And the effect of BBR to intestinal dysbacteriosis, multiple organ damages and over-activated immune system in MRL/Lpr mice.

Methods: $16 \mathrm{~S}$ high-throughput (16S rRNA) sequence, qRT-PCR and gas chromatography technology were used to determine the gut microbiota and metabolites in 104 SLE patients from Affiliated Hospital of Nantong University, China. BBR was orally treated to the MRL/Lpr mice in low, medium and high doses. After 6 weeks treatment, mice were sacrificed. Serum, faeces and organs were collected for further studies.

Results: Chinese SLE patients showed higher abundance of Bacteroidetes and lower abundance of Firmcutes. The results of qRT-PCR showed high Firmcutes/Bacteroidetes (F/B) ratio of SLE patients. The F/B ratio was negative correlated with SLE disease activity index (SLEDA) score. Almost all the tested short-chain fatty acids (SCFAs) found statistically significant results in SLE and LN (lupus nephritis) patients, especially the propanoic acid and butyric. BBR altered the relative abundance of Bacteroides and Verrucomicrobia and the butyric acid content in colon of MRL/Lpr mice. The increase of tight junction protein also indicated the gut barrier was repaired by BBR. Treg and Tfr cells in spleen and mesenteric lymph node (MLN) were increased. These results revealed a positive therapeutic effect of berberine on SLE from gut microbiota to immune status.

Conclusion: Our study highlights current status of intestinal dysbacteriosis in Chinese patients with SLE and differences in intestinal metabolites among patients with different disease states. The regulation of intestinal flora and the repairment of gut barrier by intestinal metabolites in BBR treated mice seemed to be the factor that directed the immune responses and disease outcomes. The ultimate goal of our study was to determine the beneficial effects of regulating the gut microbiota on the treatment of SLE. The application of berberine is a relatively safe and convenient way. In the coming investigations, we plan to focus on the study of berberine and its metabolites on intestinal function and systemic immunity.

\section{REFERENCES:}

[1] Guo, M. et al. Alteration in gut microbiota is associated with dysregulation of cytokines and glucocorticoid therapy in systemic lupus erythematosus. Gut microbes 11, 1758-1773, doi:10.1080/19490976.2020.1768644 (2020).

[2] Mu, Q. et al. Control of lupus nephritis by changes of gut microbiota. Microbiome 5, 73, doi:10.1186/s40168-017-0300-8 (2017).

[3] Habtemariam, S. Berberine pharmacology and the gut microbiota: A hidden therapeutic link. Pharmacological research 155, 104722, doi:10.1016/j. phrs.2020.104722 (2020).

[4] Cui, H. et al. Berberine Regulates Treg/Th17 Balance to Treat Ulcerative Colitis Through Modulating the Gut Microbiota in the Colon. Frontiers in pharmacology 9, 571, doi:10.3389/fphar.2018.00571 (2018).

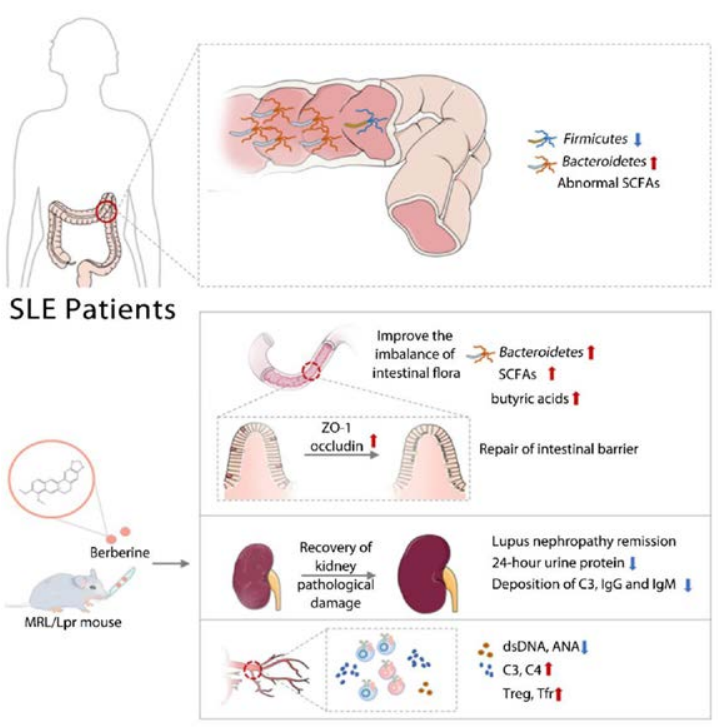

Figure 1.

Disclosure of Interests: None declared DOI: 10.1136/annrheumdis-2021-eular.3838

\section{POS0788 CIRCULATING EXOSOMES PROMOTE LUPUS NEPHRITIS IN MRL-LPR MICE}

X. Sha ${ }^{1}$, X. Ge ${ }^{1}$, Y. Jin ${ }^{1}$, T. Chen ${ }^{1}$, X. Ni ${ }^{1}$, W. Zheng ${ }^{2}$, J. Ji ${ }^{1}$, Z. Gu ${ }^{1} .{ }^{1}$ Affiliated Hospital of Nantong University, Department of Rheumatology, Nantong, China; ${ }^{2}$ Affiliated

Hospital of Nantong University, Research Center of Clinical Medicine, Nantong, China 